\title{
Disponibilidade de fósforo para a cana-de-açúcar em solo tratado com compostos orgânicos ricos em silício
}

\author{
$\overline{\text { Claudivan C. Lima }^{1}}$
}

\begin{abstract}
RESU M O
Avaliou-se a disponibilidade de $\mathrm{P}$ para a cultura da cana-de-açúcar em solo tratado com compostos orgânicos ricos em silício. Para tanto, cinco compostos foram obtidos a partir da mistura dos seguintes materiais: bagaço de cana-de-açúcar, cinzas de bagaço de cana, torta de filtro e farelo de mamona, enriquecidos com pó de rochas silicatadas. Os tratamentos, constituídos pela aplicação de 5 doses $(0$, 13, 26, 52 e 104 t ha $^{-1}$ ) dos referidos compostos +1 tratamento adicional com adubação química (N PK). Após sua aplicação amostras de solo foram coletadas e determinados P-remanescente, Si "disponível", $\mathrm{C}$ total e fracionamento das substâncias húmicas. Aos 150 dias de cultivo foram coletadas amostras da planta para análise de $\mathrm{P}$ foliar. A aplicação dos compostos possibilitou a redução da fixação de $\mathrm{P}$ pelo solo, contribuindo para a elevação da absorção deste elemento pela cultura da cana-de-açúcar; a redução da fixação de $P$ pelo solo foi atribuída ao $S i$ proveniente do pó de rochas silicatadas e às substâncias húmicas presentes nesses compostos; os compostos contendo cinza de bagaço de cana não contribuíram para a elevação dos teores de Si "disponível" no solo.
\end{abstract}

Palavras-chave: silício “disponível”, substâncias húmicas, fixação de P

\section{Availability of phosphorus for sugarcane in soil treated with silicon rich organic composts}

\begin{abstract}
Availability of $P$ for sugarcane plants was evaluated in a soil treated with organic composts rich in silicon. Five composts were formulated with following wastes: sugarcane bagasse, ashes of sugarcane bagasse, filter cake and castor bean plant residue enriched with silicon powdered rocks. The treatments consisted of five doses $\left(0,13,26,52\right.$ e $\left.104 \mathrm{t} \mathrm{ha}^{-1}\right)$ of each compost and an additional treatment with mineral fertilization (N PK). Soil samples were collected after the application of the treatments in order to carry out the following analyses: equilibrium $\mathrm{P}$, "available" $\mathrm{Si}$, total organic $\mathrm{C}$, and fractionation of the organic substances. The plant was sampled 150 days after planting for analysis of the leaf $P$. The application of composts made possible reduction of the adsorption of soil $P$, contributing to raise the absorption of this element by sugarcane crop; the reduction of the adsorption of $P$ in the soil was atributed to Si from rocks of silicon, and the humic substances from composts and the compost containing ashes of sugarcane bagasse did not contribute to elevate content of "available" Si in the soil.
\end{abstract}

Key words: "Available" Si, humic substances, adsorption of $\mathrm{P}$ 


\section{INTRODUÇÃO}

A disponibilidade de fósforo para as plantas em solos tropicais é influenciada pela adição deste elemento por meio da adubação fosfatada e regulada pelo fenômeno de sorção de $\mathrm{P}$ pelo solo. Este fenômeno ocorre na superfície dos óxidos de $\mathrm{Fe}$ e de $\mathrm{Al}$ por meio da troca de ligantes, em que grupos $\mathrm{OH}$ são substituídos por íons fosfatos da solução do solo, diminuindo sua concentração em solução (Afif et al., 1995; Andrade et al., 2003; Santos et al., 2008). Como forma de contornar o problema e possibilitar o fornecimento de $\mathrm{P}$ às plantas, tem-se sugerido a adição de fertilizantes que contenham agentes que concorrem pelos mesmos sítios de troca do fosfato (Borggaard et al., 2005; Berwanger et al., 2008), como o silício (De \& Datta, 2007; Pozza et al., 2007) e as substâncias húmicas (SH) (Lee \& Kim, 2007; Hua et al., 2008).

$\mathrm{O} \mathrm{Si}$ pode ser adsorvido às superfícies dos óxidos de $\mathrm{Fe}$, tal como o P, inclusive deslocando este elemento previamente adsorvido nas superfícies oxídicas e vice-versa (Obihara \& Russel, 1972), contribuindo, assim, com a redução do fenômeno da sorção. Tais efeitos podem ser devidos não só à competição dos ânions silicato pelos sítios de adsorção de fosfato, mas também ao aumento do $\mathrm{pH}$, uma vez que os silicatos apresentam ação corretiva da acidez dos solos (Korndörfer et al., 1999a).

Assim como o Si, as substâncias húmicas $(\mathrm{SH})$ podem atuar na redução da adsorção do P pelo solo (Aguilera et al., 1992; Lee \& Kim, 2007), competindo pelos mesmos sítios de adsorção do fosfato. A efetividade no bloqueio desses sítios varia de acordo com a taxa de mineralização das SH. Compostos orgânicos de cadeia mais simples, por exemplo, são decompostos mais rapidamente (Geelhoed et al., 1999) e moléculas com maior número de grupos funcionais, como $\mathrm{OH}$ e $\mathrm{COOH}$, são mais efetivas na competição pelos sítios de adsorção (Andrade et al., 2003).

Os resíduos provenientes da produção de biocombustíveis, como o bagaço da cana, a cinza do bagaço da cana, a torta de filtro e o farelo da mamona, podem ser empregados na formulação de compostos orgânicos e contribuir para a obtenção de fertilizantes com maior conteúdo de SH e rico em $\mathrm{Si}$, os quais podem atuar na redução da sorção de $\mathrm{P}$ em solos tropicais. A queima do bagaço da cana, que vem sendo utilizada para a produção de energia em usinas de açúcar e álcool, gera grande quantidade de cinzas no final da estação de moagem. Esta cinza, que possui teores de Si em torno de 65 a $81 \%$ pode, quando aplicada ao solo, contribuir para o aumento da produção da cana-de-açúcar, conforme observaram Pan et al. (1978), elevando em $20 \%$ sua produção.

$\mathrm{O}$ enriquecimento mineral dos resíduos de produção de biocombustíveis durante o processo de compostagem, utilizando-se pós de rocha de serpentinito e de macaxisto, pode contribuir tanto na elevação dos teores de Si nos compostos, como promover alterações na qualidade das $\mathrm{SH}$, conferindolhe um potencial diferenciado na competição por sítios de adsorção de fosfato (Lima et al., 2010).

O presente estudo teve por objetivo avaliar o efeito da aplicação de compostos orgânicos formulados com resíduos ricos em Si sobre a disponibilidade de P para a cultura da canade-açúcar.

\section{Material E MÉTODOS}

Este estudo foi realizado em casa de vegetação localizada no Departamento de Solos da Universidade Federal de Viçosa, Viçosa, Estado de Minas Gerais. Amostra de Latossolo Vermelho-Amarelo, distrófico, textura argilo arenosa proveniente do município de João Pinheiro, Estado de Minas Gerais, foi coletada na camada de 0 a $20 \mathrm{~cm}$ e passada em peneira de 2,0 mm. Determinaram-se as seguintes características químicas e físicas desse solo: $\mathrm{pH}$ em água: 4,76; $\mathrm{P}$ - Mehlich 1: $2,1 \mathrm{mg} \mathrm{dm}^{-3} ; \mathrm{K}: 122 \mathrm{mg} \mathrm{dm}^{-3}$; Ca: 0,18 $\mathrm{cmol}_{\mathrm{c}} \mathrm{dm}^{-3} ; \mathrm{Mg}: 0,06$ cmol $\mathrm{dm}^{-3} ; \mathrm{Al}: 0,20 \mathrm{cmol}_{\mathrm{c}} \mathrm{dm}^{-3} ; \mathrm{H}+\mathrm{Al}: 3,9 \mathrm{cmol}_{\mathrm{c}} \mathrm{dm}^{-3} ; \mathrm{SB}: 0,55$ cmol $\mathrm{dm}^{-3}$; t: $0,75 \mathrm{cmol}_{\mathrm{c}} \mathrm{dm}^{-3}$; T: 4,45 $\mathrm{cmol}_{\mathrm{c}} \mathrm{dm}^{-3}$; V\%:12,4; m\%:26,7; MO: 1,43 dag kg-1; P-rem: 26,3 $\mathrm{mg} \mathrm{L}^{-1}$; densidade do solo: $1,33 \mathrm{~kg} \mathrm{dm}^{-3}$; densidade das partículas: $2,68 \mathrm{~kg} \mathrm{dm}^{-3}$; teor de argila: $37 \%$ e classe textural: argilo arenosa.

Este solo foi acondicionado em recipientes de $20 \mathrm{dm}^{3}$ para, em seguida, receberem os tratamentos. Utilizaram-se cinco compostos orgânicos cujas formulação e caracterização química constam nas Tabelas 1 e 2, respectivamente; além desses tratamentos aplicou-se mais um, adicional, constituído pela recomendação de adubação convencional da cultura da cana-de-açúcar $\left(80 \mathrm{~kg} \mathrm{ha}^{-1} \mathrm{P}_{2} \mathrm{O}_{5} ; 90 \mathrm{~kg} \mathrm{ha}^{-1} \mathrm{~K}_{2} \mathrm{O}\right.$ e $\left.60 \mathrm{~kg} \mathrm{ha}^{-1} \mathrm{~N}\right)$ (AQ), conforme Korndörfer et al. (1999b). Com antecedência de 15 dias para montagem do experimento realizou-se apenas no tratamento químico adicional, calagem do solo com a mistura de carbonato de cálcio + carbonato de magnésio (4:1) de acordo com sua necessidade, utilizando-se o método da neutralização do $\mathrm{Al}^{3+}$ e da elevação dos teores de $\mathrm{Ca}^{2+}+\mathrm{Mg}^{2+}$ trocáveis (Alvarez V. \& Ribeiro, 1999). Os tratamentos foram constituídos pelo fatorial $(5 \times 5)+1$ (cinco doses: 0, 13, 26, 52 e $104 \mathrm{t} \mathrm{ha}^{-1}$, base matéria seca, de cinco compostos orgânicos distintos +1 adubação química) e foram distribuídos em blocos casualizados, com três repetições.

Os tratamentos foram incubados durante 30 dias, com umidade a $70 \%$ da capacidade de campo. Após este período amostras de solo foram coletadas para realização das análises de C orgânico total (Yeomans \& Bremner, 1988); Premanescente (Alvarez V. et al., 2000) e fracionamento da matéria orgânica em

Tabela 1. Compostos orgânicos formulados a partir da mistura de materiais orgânicos em diferentes proporções e enriquecidos com fertilizantes minerais solúveis e pós de rocha

\begin{tabular}{ccl}
\hline $\begin{array}{c}\text { Composto } \\
\text { orgânico }\end{array}$ & Proporção & \multicolumn{1}{c}{ Composição } \\
CS & $3: 3: 2$ & $\begin{array}{l}\text { bagaço de cana + cinza de bagaço } \\
\text { de cana }+ \text { esterco de galinha } \\
\text { poedeira }\end{array}$ \\
\hline SA & $3: 3: 2$ & $\mathrm{CS}+$ NPK* (N: sulfato de amônio) \\
\hline SM & $3: 3: 2$ & $\begin{array}{l}\text { CS + micaxisto + serpentinita } \\
\text { (silicato de magnésio) - em pó }\end{array}$ \\
\hline TF & $\left.2: 5 \mathrm{~kg} \mathrm{Mg}^{-1}\right)$ & bagaço de cana + torta de filtro \\
\hline $\mathrm{M}-\mathrm{G}$ & $2: 1$ & $\begin{array}{l}\text { bagaço de cana + farelo de } \\
\text { mamona }\end{array}$ \\
\hline
\end{tabular}

* 0 enriquecimento do compos to com NPK foi procedido de modo que na aplicação da dose de 20 t ha ${ }^{-1}$ do composto contivesse a dose de fertilizante recomendada para a cana-de-açúcar que,

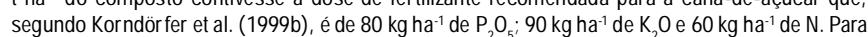
este cálculo considerou-se que a mistura de resíduos orgânicos, após compostada, teria redução de $2 / 3$ do volume inicial 
Tabela 2. Composição química de compostos orgânicos obtidos com diferentes materiais e enriquecimento mineral

\begin{tabular}{|c|c|c|c|c|c|}
\hline Característica & CS & SA & SM & TF & M-G \\
\hline $\mathrm{pH}-\mathrm{CaCl}_{2}{ }^{1 /}$ & 7,24 & 6,28 & 7,50 & 5,88 & 5,76 \\
\hline $\mathrm{Ca}^{2+}\left(\mathrm{g} \mathrm{kg}^{-1}\right)^{21}$ & 71,13 & 69,51 & 70,30 & 22,15 & 13,16 \\
\hline $\mathrm{Mg}^{2+}\left(\mathrm{g} \mathrm{kg}^{-1}\right)^{2 /}$ & 5,59 & 5,13 & 21,24 & 1,94 & 5,81 \\
\hline $\mathrm{K}\left(\mathrm{g} \mathrm{kg}^{-1}\right)^{2 !}$ & 110,16 & 96,30 & 204,70 & 478,49 & 76,24 \\
\hline $\mathrm{Al}^{3+}\left(\mathrm{g} \mathrm{kg}^{-1}\right)^{2 /}$ & 9,77 & 7,96 & 13,35 & 49,23 & 6,39 \\
\hline$P\left(g_{k g}^{-1}\right)^{2 !}$ & 9,93 & 11,50 & 9,05 & 6,54 & 5,96 \\
\hline $\mathrm{S}\left(\mathrm{g} \mathrm{kg}^{-1}\right)^{2}$ & 2,94 & 5,59 & 2,59 & 3,48 & 3,44 \\
\hline $\mathrm{Fe}\left(\mathrm{g} \mathrm{kg}^{-1}\right)^{2 !}$ & 7,20 & 5,82 & 12,67 & 29,69 & 4,41 \\
\hline $\mathrm{Cu}\left(\mathrm{mg} \mathrm{kg}^{-1}\right)^{2 /}$ & 50,08 & 57,46 & 58,44 & 48,16 & 36,68 \\
\hline $\mathrm{Zn}\left(\mathrm{mg} \mathrm{kg}^{-1}\right)^{2 /}$ & 326,29 & 284,64 & 249,66 & 145,52 & 158,95 \\
\hline$B\left(\mathrm{mg} \mathrm{kg}^{-1}\right)^{2}$ & nd & nd & nd & 4,21 & nd \\
\hline$M_{0}\left(\mathrm{mg} \mathrm{kg}^{-1}\right)^{21}$ & 1,38 & 0,25 & nd & nd & nd \\
\hline $\mathrm{Mn}\left(\mathrm{mg} \mathrm{kg}^{-1}\right)^{21}$ & 472,95 & 394,51 & 503,63 & 892,62 & 330,24 \\
\hline Si Total $\left(\mathrm{mg} \mathrm{kg}^{-1}\right)$ & 25,06 & 36,65 & 40,85 & 12,67 & 5,57 \\
\hline C Total $\left(\mathrm{g} \mathrm{kg}^{-1}\right)$ & 141,51 & 162,34 & 85,28 & 277,72 & 309,77 \\
\hline $\mathrm{N}$ Total $\left(\mathrm{g} \mathrm{kg}^{-1}\right)$ & 8,90 & 11,20 & 10,30 & 18,70 & 27,90 \\
\hline Relação C/N & 15,90 & 14,50 & 8,30 & 14,80 & 11,10 \\
\hline FAH (dag kg $\left.{ }^{-1}\right)$ & 0,98 & 0,79 & 0,92 & 1,89 & 2,87 \\
\hline FAF $\left(\right.$ dag $\left.\mathrm{kg}^{-1}\right)$ & 0,68 & 0,32 & 0,49 & 1,77 & 1,22 \\
\hline $\mathrm{SH}\left(\operatorname{dag~kg}^{-1}\right)$ & 1,66 & 1,11 & 1,41 & 3,66 & 4,09 \\
\hline
\end{tabular}

1/ Relação sólido-solução 1:5; 2/ Digestão nitroperclórica, leitura em ICP-OES; FAH: fração ácidos húmicos; FAF: fração ácidos fúlvicos; SH: substâncias húmicas; CS: composto simples; SA: CS enriquecido com NPK (N: sulfato de amônio); SM: CS enriquecido com pó de rocha serpentinito + micaxisto; TF: torta de filtro + bagaço de cana; M-G: farelo de mamona + bagaço de cana; nd: não detectado

ácidos húmicos, fúlvicos e humina, de acordo com a International Humic Substaces Society - IHSS (Hayes et al., 1989), determinando-se o teor de C nessas frações; silício "disponível" extraído com solução de cloreto de cálcio $10 \mathrm{mmol} \mathrm{L}^{-1}$, segundo Korndörfer et al. (2004); em seguida, foram plantados os toletes pré-geminados de cana-de-açúcar (Saccharum officinarum L.), variedade RB867515; aos 150 dias após o plantio amostras da folha +3 foram coletadas para avaliação do teor de $\mathrm{P}$, por meio de espectrometria de emissão óptica em plasma induzido (ICPOES), após secagem a $65^{\circ} \mathrm{C}$ e moagem da amostra e digestão nitroperclórica.

Os dados obtidos foram submetidos à análise de variância, de regressão e correlação linear simples (Correlação de Pearson), utilizando-se o programa SAEG (Sistema de Análises Estatísticas e Genéticas), da Universidade Federal de Viçosa.

\section{RESULTADOS E DISCUSSÃO}

\section{Silício "disponível”}

Os teores de $\mathrm{Si}$ "disponível” no solo apresentaram comportamento linear com o aumento das doses dos compostos nos tratamentos SM, TF e M-G, enquanto se verificou no tratamento SA, comportamento quadrático (Figura 1A). No solo tratado com compostos TF e M-G se observaram, embora esses apresentassem os menores quantitativos de Si total (Tabela 2), os maiores teores do $\mathrm{Si}$ "disponível" com incremento linear decorrente do aumento das doses. Tal disponibilidade pode ser atribuída ao processo de mineralização do bagaço da cana durante o processo de compostagem que, provavelmente, possibilitou a solubilização do Si contido neste material, estando prontamente disponível quando da sua aplicação ao solo. Já os compostos CS e SA, embora apresentem teores de Si total mais elevados (Tabela 2) em virtude da presença de sílica gel contida no bagaço de cana (BC) (Savat et al., 1999) e de sílica cristalina presente no quatzo da cinza de bagaço de cana (CBC) (Teixeira et al., 2008), sofreram pouca influência das doses empregadas. Essas diferentes formas de silício se combinaram, provavelmente, formando ácido polissilíssico, durante o processo de compostagem. Por esta razão, verificouse diminuição dos teores de Si “disponível” quando da aplicação dos compostos CS e SA no solo, haja vista o ácido polissilícico ser uma forma indisponível deste elemento (Matichenkov \& Snyder, 1996).
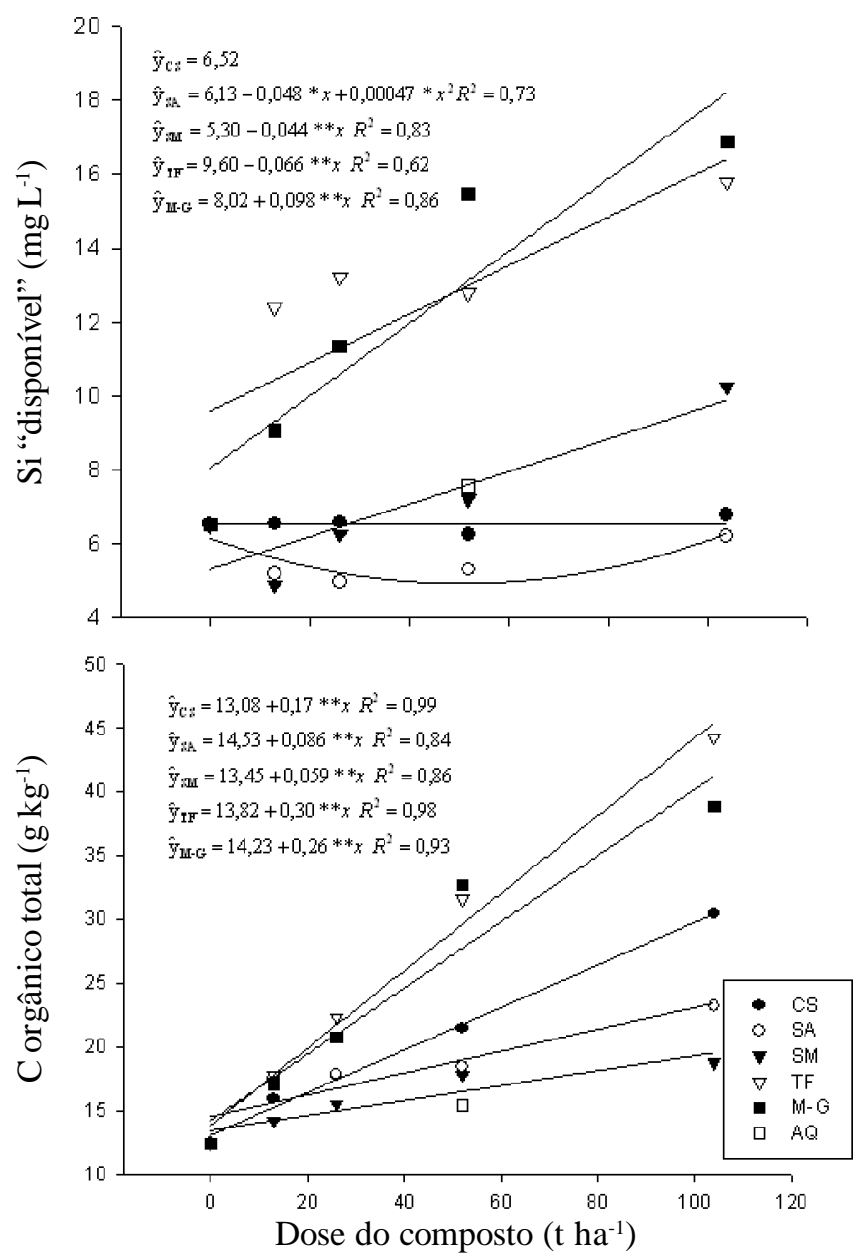

CS: composto simples; SA: CS enriquecido com NPK, sendo N: sulfato de amônio; SM: CS enriquecido com pó de rocha serpentinito + micaxisto; TF: torta de filtro + bagaço de cana; M$\mathrm{G}$ : farelo de mamona + bagaço de cana; AQ: adubação química (NPK); *, **, significância de 5 e $1 \%$ de probabilidade, respectivamente, pelo teste $t$

Figura 1. Teores de silício "disponível" (A) e teores de $C$ orgânicos (B) de solo submetido à aplicação de doses crescentes de diferentes compostos orgânicos

Já no solo tratado com o composto SM o teor de $\mathrm{Si}$ "disponível" foi superior ao do tratamento $\mathrm{AQ}$ a partir da dose de $56 \mathrm{t} \mathrm{ha}^{-1}$. Este elemento estava presente no BC e na CBC e também nos pós de rocha de serpentinito e de micaxisto contidos no composto SM. Esses pós de rocha parecem ter sofrido alguma solubilização durante o processo de compostagem, denotada pelo aumento linear do Si "disponível" com o aumento da dose aplicada, sobrepujando o efeito 
negativo da combinação das distintas formas de Si presentes nos $\mathrm{BC}$ e CBC.

\section{Carbono orgânico total e $\mathrm{C}$ das frações ácidos húmicos e fúlvicos}

A adição de doses crescentes dos compostos orgânicos elevou os teores de C orgânico (Figuras 1B). Essas elevações foram tanto maiores quanto maior o teor de $\mathrm{C}$ orgânico nos materiais (Tabela 1).

Os teores de $\mathrm{C}$ da fração ácido húmico $(\mathrm{FAH})$, fração ácido fúlvico (FAF) e fração humina (FHU) apresentaram crescimento linear com o aumento das doses dos compostos sendo a FHU a que apresentou teores mais elevados (Figura 2). Verificou-se predominância da FAF em relação aos FAH $(\mathrm{FAH} / \mathrm{FAF}<1)$ nos tratamentos CS, SA, SM e M-G. Esta mesma tendência foi
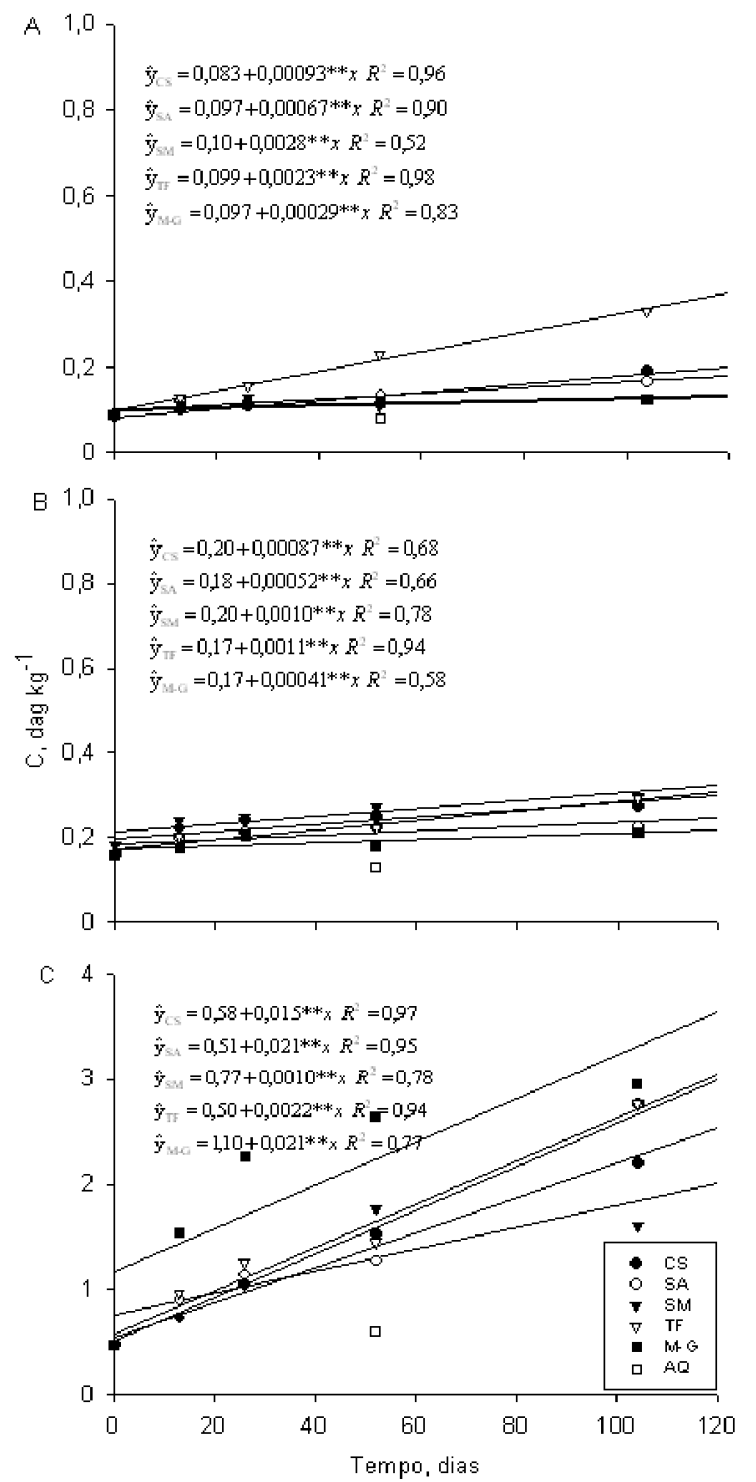

CS: composto simples; SA: CS enriquecido com NPK, sendo N: sulfato de amônio; SM: CS enriquecido com pó de rocha serpentinito + micaxisto; TF: torta de filtro + bagaço de cana; MG: farelo de mamona + bagaço de cana; AQ: adubação química (NPK); *, **, significância de 5 e $1 \%$ de probabilidade, respectivamente, pelo teste $t$

Figura 2. Teores de $C$ das frações ácido húmico $(A)$, ácido fúlvico $(B)$ e humina $(C)$ de solo cultivado com cana-de-açúcar e submetido à aplicação de doses crescentes de diferentes compostos orgânicos observada no tratamento TF na aplicação das primeiras doses do referido composto, sendo que esta relação se inverteu com $\mathrm{o}$ aumento das doses. $\mathrm{O}$ teor de $\mathrm{C}$ da FAF apresentou pequena diferença entre os tratamentos enquanto o $\mathrm{C}$ da FAH, do tratamento TF, apresentou teores mais elevados, destacandose dos demais tratamentos. Esses resultados não se devem apenas aos aspectos quantitativos de FAH deste composto (Tabela 2), mas também às características qualitativas desta fração (Lima et al., 2010).

\section{Fósforo remanescente}

Em geral, o P remanescente apresentou valores crescentes com o aumento da dose do composto (Figura 3), resultado que evidencia o efeito do composto orgânico em diminuir a precipitação/adsorção de $\mathrm{P}$ pelo solo, em todos os tratamentos, denotado pela correlação positiva entre $\mathrm{P}$ remanescente e $\mathrm{C}$ total $\left(\mathrm{r}=0,78^{* *}\right)$. Contudo, analisando-se a correlação dentro de cada composto do P remanescente com a FAH [SA ( $\mathrm{r}=$ $\left.0,92^{* *}\right), \mathrm{TF}\left(\mathrm{r}=0,91^{* *}\right), \mathrm{CS}\left(\mathrm{r}=0,88^{* *}\right), \mathrm{M}-\mathrm{G}\left(\mathrm{r}=0,75^{* *}\right)$ e SM $\left.\left(\mathrm{r}=0,69^{* *}\right)\right]$ e com a FAF $\left[\mathrm{CS}\left(\mathrm{r}=0,93^{* *}\right), \mathrm{TF}\left(\mathrm{r}=0,92^{* *}\right), \mathrm{SM}\right.$ $(\mathrm{r}=0,90 * *), \mathrm{SA}\left(\mathrm{r}=0,88^{* *}\right)$ e M-G $\left.(\mathrm{r}=0,80 * *)\right]$, verificaram-se, em geral, correlações mais significativas, comparativamente com o $\mathrm{C}$ total, devido à ação direta das $\mathrm{SH}$ na formação de complexos organo-metálicos com os íons $\mathrm{Fe}$ e $\mathrm{Al}$, em várias faixas de $\mathrm{pH}$, que podem impedir a precipitação do fosfato com tais íons. Além disso, as SH podem bloquear os sítios de adsorção de fosfato da matriz do solo e formar "capas" ou "superfícies protetoras" nos oxi-hidróxidos de Fe e Al (Mesquita Filho \& Torrent, 1993), contribuindo, sobremaneira, com a redução da forte adsorção de P (Bhatti et al., 1998; Borggaard et al., 2005) e aumentando, consequentemente, a disponibilidade deste elemento às plantas. Guarçoni \& Mendonça (2003) também verificaram elevação linear do teor de $\mathrm{P}$ remanescente de solos tropicais com a aplicação de doses crescentes de composto até $40 \mathrm{t} \mathrm{ha}^{-1}$.

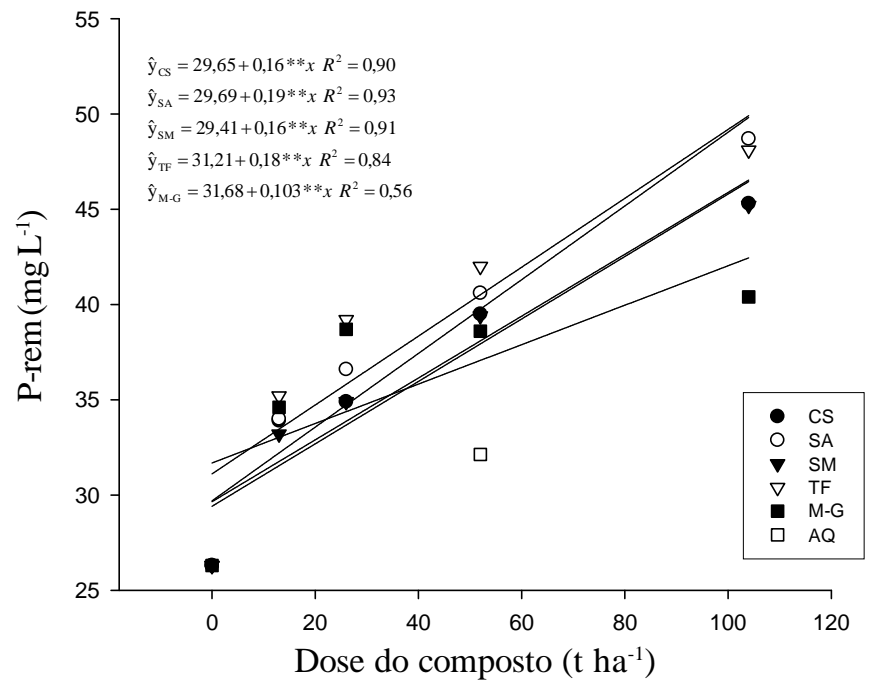

CS: composto simples; SA: CS enriquecido com NPK, sendo N: sulfato de amônio; SM: CS enriquecido com pó de rocha serpentinito + micaxisto; TF: torta de filtro + bagaço de cana; $M$ $\mathrm{G}$ : farelo de mamona + bagaço de cana; AQ: adubação química (NPK); *, **, significância de 5 e $1 \%$ de probabilidade, respectivamente, pelo teste $t$

Figura 3. Valores de $\mathrm{P}$ remanescente (P-rem) de solo submetido à aplicação de doses crescentes de diferentes compostos orgânicos 
O Si solúvel pode ter influenciado a dinâmica de P no solo, observando-se algumas correlações entre o Si "disponível” e o $\mathrm{P}$ remanescente nos tratamentos $\mathrm{SM}\left(\mathrm{r}=0,70^{* *}\right)$, TF $(\mathrm{r}=$ $0,94 * *)$ e M-G $\left(\mathrm{r}=0,78^{* *}\right)$. O Si contido no composto SM proveniente dos pós de rocha, provavelmente promoveu bloqueio nos sítios de adsorção de fosfatos, assim como as SH presentes neste composto, haja vista ambos apresentarem correlações positivas com o $\mathrm{P}$ remanescente, diferentemente dos outros tratamentos contendo CBC.

\section{Fósforo foliar}

O teor de $\mathrm{P}$ foliar da cana-de-açúcar apresentou diferenças entre os tratamentos e entre as doses de compostos utilizadas (Figura 4). Os tratamentos com compostos obtiveram, em média, teor foliar deste nutriente superior ao do tratamento químico (AQ). Com teor de P foliar de 0,17 dag $\mathrm{kg}^{-1}$, o tratamento AQ apresentou teor inferior ao encontrado por Martinez et al. (1999), que foi de 0,21 a $0,25 \mathrm{dag} \mathrm{kg}^{-1}$. Dentre os compostos orgânicos os tratamentos que mais se destacaram foram o TF e o M-G apresentando, como tendência geral, maiores teores foliares de $\mathrm{P}$ com o aumento das doses aplicadas. A despeito dos compostos TF e M-G conterem menores teores de $\mathrm{P}$ total (Tabela 1), eles conferiram maior teor de $\mathrm{P}$ foliar à cultura da cana-de-açúcar, cujo resultado pode ser atribuído à elevação dos teores de Si "disponível" no solo quando da aplicação desses compostos e também ao fato dos mesmos apresentarem maiores quantitativos de $\mathrm{SH}$ comparativamente aos demais o que provavelmente, resultou na redução da fixação do $\mathrm{P}$ pelo solo, tornando este elemento mais prontamente disponível à cultura da cana-de-açúcar.

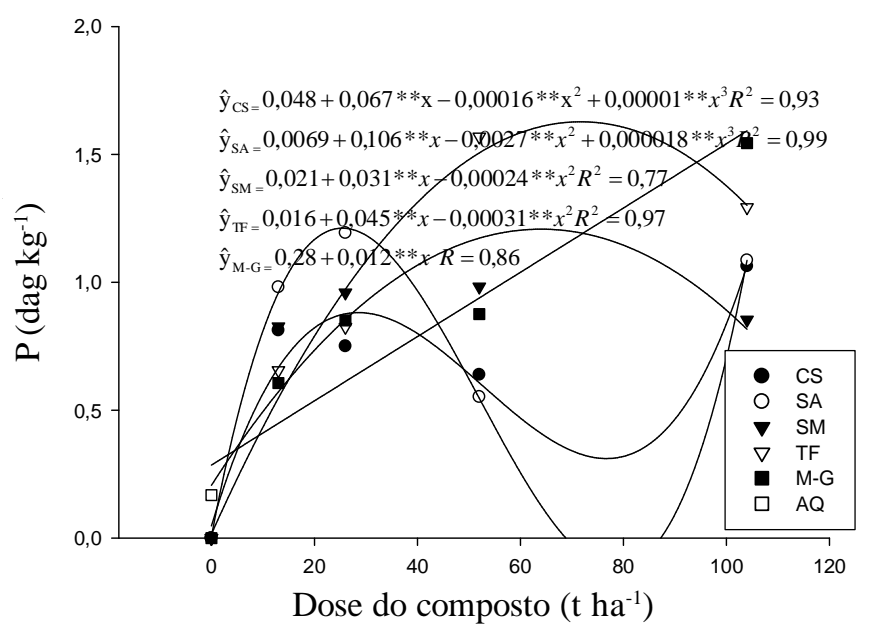

CS: composto simples; SA: CS enriquecido com NPK, sendo N: sulfato de amônio; SM: CS enriquecido com pó de rocha serpentinito + micaxisto; TF: torta de filtro + bagaço de cana; M$\mathrm{G}$ : farelo de mamona + bagaço de cana; AQ: adubação química (NPK); *, **, significância de 5 e $1 \%$ de probabilidade, respectivamente, pelo teste $t$

Figura 4. Teores de $P$ foliar da cana planta, variedade RB867515, com cinco meses de idade, cultivada em solo submetido à aplicação de doses crescentes de diferentes compostos orgânicos

Orlando Filho (1978) encontrou, em cana de seis meses, variedade CB-453, teor foliar de P de 0,08 dag $\mathrm{kg}^{-1}$, inferior ao teor apresentado no presente estudo em cana da variedade RB867515, ao cabo de cinco meses de cultivo, enquanto
Mendes (2006), avaliando esta mesma variedade de cana, obteve teor de $0,15 \mathrm{dag} \mathrm{kg}^{-1}$ aos 10 meses de cultivo, também inferior à média dos teores de $\mathrm{P}$ foliar dos tratamentos. Essas diferenças podem estar relacionadas às características próprias de cada variedade, como observou Mendes (2006), acrescido do fato das plantas do presente estudo serem relativamente jovens e, normalmente, ocorrer diminuição do teor de minerais com a idade da planta, em função da translocação de nutrientes das folhas para outras partes da planta, como o colmo (Orlando Filho, 1978).

$\mathrm{O}$ teor de $\mathrm{P}$ foliar se correlacionou positivamente com o seu respectivo $\mathrm{P}$ remanescente do solo $(\mathrm{r}=0,79 * *)$, indicando que a dinâmica de decomposição da $\mathrm{MO}$ atendeu prontamente à demanda da cultura, embora a análise foliar, para avaliar a necessidade de nutrientes para a cana-de-açúcar apresente, em geral, baixa sensibilidade, conforme observado por Rossetto et al. (2004).

\section{CONCLusÕES}

1. A aplicação do composto orgânico possibilitou a redução da fixação de $\mathrm{P}$ pelo solo, contribuindo para a elevação da absorção do mineral pela cultura da cana-de-açúcar;

2. A redução da fixação de P pelo solo quando da aplicação do composto orgânico, pode ser atribuída às substâncias húmicas presentes nos compostos;

3. O Si proveniente dos pós de rocha de serpentinito e micaxisto presente no composto SM, também contribuiu para a redução da fixação de $\mathrm{P}$ pelo solo possibilitando aumento da absorção de P, pela cultura da cana-de-açúcar;

4. Os compostos contendo cinza de bagaço de cana não contribuíram para a elevação dos teores de Si "disponível" no solo, não influenciando, portanto, a redução da fixação de P.

\section{AgRAdECIMENTOS}

O autor agradece aos professores Eduardo de Sá Mendonça e Ivo Ribeiro da Silva, ambos do Departamento de Solos da Universidade Federal de Viçosa, pelas relevantes contribuições na discussão dos resultados, e ao CNPq e à FAPEAL, pelo suporte financeiro visando à realização desta pesquisa.

\section{LITERATURA CITADA}

Afif, E.; Barrón, V.; Torrent, J. Organic matter delays but does not prevent phosphate sorption by cerrado soils from Brazil. Soil Science, v.159, p.207-211, 1995.

Aguilera, M. S.; Pino, I. U.; Reyes, C. P., Caiozzi, M. C. Effect of organic matter on soil availability of phosphorus, iron, cooper and zinc in Osborne. Agricultura Técnica, v.52, p.423425, 1992.

Alvarez V., V. H.; Novais, R. F.; Dias, L. E.; Oliveira, J. A. Determinação do uso do fósforo remanescente. Boletim Informativo da Sociedade Brasileira de Ciência do Solo, v.25, p.27-32, 2000 . 
Alvarez V., V. H.; Ribeiro, A. C. Calagem. In: Ribeiro, A. C.; Guimarães, P. T. G. Alvarez V., V. H. (ed.). Recomendações para o uso de corretivos e fertilizantes em Minas Gerais. $5^{\text {a }}$ aproximação. Viçosa: Comissão de Fertilidade do Solo do Estado de Minas Gerais, 1999. Cap.8, p.43-60.

Andrade, F. V.; Mendonça, E. S.; Alvarez V., V. H.; Novais, R. F. Adição de ácidos orgânicos e húmicos em Latossolo e adsorção de fosfato. Revista Brasileira de Ciência do Solo, v.27, p.1003-1011, 2003.

Berwanger, A. L.; Ceretta, C. A.; Santos, D. R. Alterações no teor de fósforo no solo com aplicação de dejetos líquidos de suínos. Revista Brasileira de Ciência do Solo, v.32, p.25252532, 2008.

Bhatti, J. S.; Comeford, N. B.; Johnston, C. T. Influence of oxalate and soil organic matter on sorption and desorption of phosphate onto a Spodic horizon. Soil Science Society America of Journal, v.62, p.1089-1095, 1998.

Borggaard, O. K.; Raben-Lange, B.; Gimsing A. L.; Strobel, B. W. Influence of humic substances on phosphate adsorption by aluminium and iron oxides. Geoderma, v.127, p.270-279, 2005.

De, N.; Datta, S. C. Relationship between phosphorus sorption and soil acidity as affected by bicarbonate and silicate ions. Communications in Soil Science and Plant Analysis, v.38, p.679-694, 2007.

Geelhoed, J. S.; van Riemsdijk, W. H.; Findenegg, G. R. Simulation of the eeffect of citrate exudation from roots on the plant availability of phosphate adsorbed on goethite. European Journal of Soil Science, v.50, p.379390, 1999.

Guarçoni, M.; Mendonça, E. S. Capacidade tampão de pH do solo e disponibilidade de fósforo pela adição de composto orgânico. Magistra, v.15, 2003.

Hayes, M. H.; Mccarthy, P.; Malcolm, R. L.; Swift, R. S. Strutures of humic substances: The emergence of forms. In: Hayes, M. H.; McCarthy, P.; Malcolm, R. L.; Swift, R. S. Humic substance II: In search of structure: Setting the scene. New York: John Wiley \& Sons, 1989. Cap.1, p.3-31.

Hua, Q. X.; Li, J. Y; Zhou, J. M.; Wang, H. Y.; Du, C. W.; Chen, X.Q. Enhancement of phosphorus solubility by humic substances in Ferrosols. Pedosphere, v.18, p.533538, 2008.

Korndörfer, G. H.; Arantes, V. A.; Corrêa, G. F.; Snyder, G.H. Efeito da aplicação de silicato de cálcio em solos cultivados com arroz de sequeiro. Revista Brasileira de Ciência do Solo, v.23, p.623-629, 1999a.

Korndörfer, G. H.; Pereira, H. S.; Nolla, A. Análise de silício: Solo, planta, fertilizante. 1.ed., Uberlândia: UFU, 2004. 7p. Boletim técnico, 2.

Korndörfer, G. H.; Ribeiro, A. C.; Andrade, L. A. B. Cana-deaçúcar. In: Ribeiro, A. C.; Guimarães, P. T. G; Alvarez V., V. H. (ed.). Recomendações para o uso de corretivos e fertilizantes em Minas Gerais. 5a aproximação. Viçosa: Comissão de Fertilidade do Solo do Estado de Minas Gerais, 1999b. Cap.18, p.285-288.
Lee, Y. B.; Kim, P. J. Reduction of phosphate adsorption by ion competition with silicate in soil. Korean Journal of Environmental Agriculture, v.26, p.286-293, 2007.

Lima, C. C.; Mendonça, E. S.; Roig, A. Contribution of humic substances from different composts on the content of humin in a tropical soil. Revista Brasileira de Ciência do Solo, v.34, p.1041-1048, 2010.

Martinez, H. E. P.; Carvalho, J. G.; Souza, R. B. Diagnose foliar. In: Ribeiro, A. C.; Guimarães, P. T. G.; Alvarez V., V. H. (ed.). Recomendações para o uso de corretivos e fertilizantes em Minas Gerais. 5a aproximação. Viçosa: Comissão de Fertilidade do Solo do Estado de Minas Gerais, 1999. Cap.17, p.285-288.

Matichenkov, V. V.; Snyder, G. H. The mobile silicon compounds in some South Florida soils. Eurasian Soil Science, v.12, p.1165-1173, 1996.

Mendes, L. C. Eficiência nutricional de cultivares de cana-deaçúcar. Viçosa: UFV, 2006. 46p. Dissertação Mestrado

Mesquita Filho, M. V.; Torrent, J. Phosphate sorption as related to mineralogy of a hydrosequence of soil from de cerrado region (Brazil). Geoderma, v.58, p.107-123, 1993.

Obihara, C. H.; Russel, E. W. Specific adsorption of the silicate and phosphate by soils. European Journal of Soil Science, v.23, p.105-117, 1972.

Orlando Filho, J. Absorção dos macronutrientes pela cana-deaçúcar (Saccharum spp.), variedade CB41-76 em três grandes grupos de solos do Estado de São Paulo. Piracicaba: ESALQ/USP, 1978. 154p. Tese Doutorado

Pan, Y. C.; Edw, K. L.; Ling, S. H. The effect of bagasse furnance ash on the growth of plant cone. In: Congress International Society of Super Tech, 16, São Paulo, 1978. Proceedings... São Paulo: Society of Super Tech, v.2, 1978, p.883-889.

Pozza, A. A. A.; Curi, N.; Costa, E. T. S.; Guilherme, L. R. G.; Marques, J. J. G. S. M.; Motta, P. E. F. Retenção e dessorção competitivas de ânions inorgânicos em gibbsita natural de solo. Pesquisa Agropecuária Brasileira, v.42, p.1627-1633, 2007.

Rossetto, R.; Spironello, A.; Cantarella, H.; Quaggio, J. A. Calagem para a cana-de-açúcar e sua interação com a adubação potássica. Bragantia, v.63, p.105-119, 2004.

Santos, D. R.; Gatiboni, L.C.; Kaminski, J. Fatores que afetam a disponibilidade do fósforo e o manejo da adubação fosfatada em solos sob sistema plantio direto. Ciência Rural, v.38, p.576-586, 2008.

Savat, N. K.; Korndöfer, G. H.; Snyder, G. H.; Datnoff, L. E. Silicon nutrition and sugarcane production: A review. Journal of Plant Nutrition, v.12, p.1853-1903, 1999.

Teixeira, S. R.; Souza, A. E.; Santos, G. T. A.; Peña, A. F. V.; Miguel, A. G. Sugarcane bagasse ash as a potential quartz replacement in red ceramic. Journal of the American Ceramic Society, v.91. p.1883-1887, 2008.

Yeomans, J. C.; Bremner, J. M. A rapid and precise method for routine determination of organic carbon in soil. Communications in Soil Science and Plant Analysis, v.19, p.1467-1476, 1988. 